AperTO - Archivio Istituzionale Open Access dell'Università di Torino

\title{
Role of explicit verbal information in conditioned analgesia
}

\section{This is a pre print version of the following article:}

Original Citation:

\section{Availability:}

This version is available http://hdl.handle.net/2318/1731492

since 2020-02-26T18:05:23Z

Published version:

DOI:10.1002/ejp.579

Terms of use:

Open Access

Anyone can freely access the full text of works made available as "Open Access". Works made available under a Creative Commons license can be used according to the terms and conditions of said license. Use of all other works requires consent of the right holder (author or publisher) if not exempted from copyright protection by the applicable law. 


\section{The role of explicit verbal information in conditioned analgesia}

Running head: Explicit verbal information in conditioned analgesia

Elisa Carlino, ${ }^{1,2}$, Diana M.E. Torta ${ }^{3,4}$, Alessandro Piedimonte ${ }^{3}$, Elisa Frisaldi ${ }^{1,2}$, Sergio Vighetti ${ }^{1}$, Fabrizio Benedetti ${ }^{1,2}$

${ }^{1}$ Department of Neuroscience, University of Turin Medical School, Turin, Italy

${ }^{2}$ National Institute of Neuroscience, Turin, Italy

${ }^{3}$ Department of Psychology, University of Turin, Turin, Italy

${ }^{4}$ CCS fMRI-Brain Connectivity and Complex Systems Unit, Koelliker Hospital, Turin, Italy

\section{Corresponding author:}

Elisa Carlino

Dipartimento di Neuroscienze, Università di Torino, Corso Raffaello 30, 10125, Torino, Italy Phone: +39011 6708491

Fax: +390116708174

e-mail: elisa.carlino@unito.it - elisa.carlino@gmail.com 


\section{Category}

Original article

\section{Acknowledgements}

This work was supported by grants from Volkswagen Foundation, NH-BEE Project, Compagnia di San Paolo.

\section{Conflict of interest}

None declared.

\section{Bulleted statements}

* There is a lively debate as to whether conditioning is an automatic process or rather it is cognitively mediated.

* This debate is thriving in the field of pain within the context of conditioned placebo analgesia.

* According to our study, pairing a visual cue with different pain stimulus intensities (conditioning procedure) is not sufficient per se to produce a conditioned analgesic response.

* What matters is the explicit verbal information about the cue itself, which indicates a crucial cognitive component in conditioned analgesia.

* These data may have important implications in clinical practice, for they show that verbal communication and interaction between health professionals and patients are essential elements in the therapeutic outcome. 


\begin{abstract}
BACKGROUND: The exact role of expectation in conditioned analgesia is still elusive as it is not clear whether conditioning is an automatic process or rather it is cognitively mediated. This study is aimed at understanding the role of explicit verbal information in conditioned analgesia. METHODS: Two groups of healthy subjects received a conditioning procedure whereby two visual cues were paired with increase and decrease in stimulus intensity. In the "conditioning/verbal information” group (VER), subjects were informed about the meaning of the cues, whereas no information was given to the second group (noVER). After two conditioning blocks, an evocation session was run in which the stimulus intensity was the same, irrespective of the cues. Pain perception was assessed according to a Numerical Rating Scale (NRS) from 0 (no pain) to 10 (maximal pain). The N2-P2 component of laser-evoked potentials (LEP) was used as an index of pain measure. RESULTS: In the evocation session, only the VER group reported a decrease in pain rating and LEP amplitude when the - cues were presented, suggesting that the visual-analgesic association does not occur without explicit verbal information. CONCLUSIONS: In line with the cognitive theory of conditioning, our results indicate that just pairing a cue with different pain stimulus intensities is not sufficient per se to produce a learning process. What matters is the informational cognitive content of the cue, i.e. the meaning assigned to the cue itself. These findings may help understand the mechanisms of conditioned analgesia and more in general of learning.
\end{abstract}

Key words: conditioning, analgesia, laser-evoked potentials, pain, learning 


\section{Introduction}

Pain is a sensory and emotional experience that is influenced by different factors, such as emotions (Villemure and Bushnell, 2002;2009) attention (Bushnell et al., 2013) and anticipation of pain (Arntz et al 1996). Learning plays an important role as well. For example, conditioned analgesia is the reduction of pain perception after the re-exposure to a context or cue previously paired with analgesia (Wickramasekera, 1980; Ader, 1997; Siegel, 2002). It is due to an associative learning process in which two stimuli, the unconditioned stimulus (US) and the conditioned stimulus (CS), are repeatedly paired. Whereas the US (e.g. stimulus intensity reduction or administration of a painkiller) induces per se an unconditioned response (UR, i.e. reduction in pain perception), the CS is a neutral stimulus with no specific analgesic effect (e.g., a visual cue), After an acquisition phase in which the US is repeatedly paired with the CS, in the subsequent evocation phase the CS alone is capable of evoking the analgesic response (conditioned response, CR) (Pavlov, 1927; Fanselow, 1998). Conditioned analgesia has been studied both in animals (Ross, 1986; Matzel and Miller, 1987; Harris and Westbrook, 1996; Siegel, 2002; Guo et al., 2010) and in humans (Voudouris et al., 1985,1990; Watson et al., 2009; Lui et al., 2010).

Today there is still a lively debate as to whether conditioning is an automatic process or rather it is cognitively mediated. This debate is thriving in the field of pain within the context of conditioned placebo analgesia (Carlino et al., 2011). Whereas Voudouris et al. (1985;1990) showed that a conditioning procedure leads to a conditioned placebo analgesic response, Mongomery \& Kirsch, (1997) and Benedetti et al. (2003) demonstrated that this conditioned placebo analgesia was actually mediated by expectation. Also, it has been reported that several brain regions, such as the orbitofrontal cortex and the ventral striatum, are activated in relation to expectations and anticipatory processes (Atlas et al., 2010). These results suggest that an important top-down cognitive component plays a crucial role in conditioned analgesia, and are in line with the cognitive theories of conditioning (Kirsch et al., 2004; Reiss, 1980; Tolman, 1932). However, the exact role 
of cognition in conditioned analgesia is still elusive, thus requiring further investigation at the empirical level.

On the basis of these considerations, in the present study we investigated whether the USCS pairing is sufficient to produce conditioned analgesia or rather verbal information about the meaning of the CS is necessary combining. To do this, we used both a psychophysical approach with pain rating scales and a neurophysiological approach with laser-evoked potentials (LEP). In particular, we analyzed the N2-P2 complex, which represents the largest LEP response with peaks at approximately 200-350 ms after painful stimulation (Bromm and Treede, 1984). This complex is modulated by expectation in a model of conditioned analgesia (Colloca et al., 2008).

\section{Methods}

\section{Subjects}

A total of 34 healthy right-handed volunteers (20 males, 14 females, age $=23 \pm 1.9$ ), recruited from the student population of the University of Turin, were engaged in the study after signing a written informed consent form in which the experimental procedure was described in detail. Participants were also informed that they were taking part in a study investigating pain perception in healthy volunteers. Each subject underwent a clinical screening aimed to rule out the consumption of medications (in particular painkillers) and caffeine beverages in the previous 12 hours. All the experimental procedures were conducted according to the policies and ethical principles of the Declaration of Helsinki. The study was approved by the local ethics committee.

\section{EEG recording and laser stimulation}

Participants sat on a comfortable chair and the electroencephalogram (EEG) was recorded from 19 scalp locations using a 19-lead EEG (Galileo; EBNeuro S.p.A). The electrodes were applied to the scalp in accordance with the 10-20 international system (Fz, F1, F2, F3, F4, F7, F8, C3, C4, Cz, P3, P4, Pz, T3, T4 T5, T6, O1, O2) with linked common ears reference. Impedance was less than $2 \mathrm{~K} \Omega$ 
in each active lead. Data were collected and digitized at a sampling rate of $512 \mathrm{~Hz}$. The electrooculogram (EOG) was recorded using two surface electrodes placed above and below the right eye. Laser stimulations were delivered by a $\mathrm{CO}_{2}$ laser (Neurolas, El.En, Florence, Italy) on the dorsum of the dominant hand (right in all participants) on a squared area $(5 \times 5 \mathrm{~cm})$ defined prior to the beginning of the experimental sessions. The stimulation site was visualized by a He-Ne laser beam and shifted between two successive stimuli in order to avoid sensitization or habituation effects and skin damage. Beam diameter was $3 \mathrm{~mm}$, wave length $10.6 \mu \mathrm{m}$ and pulse duration $15 \mathrm{~ms}$ (Truini et al., 2005). Both subject and experimenter wore protective eye goggles.

A total of 72 laser stimuli, divided into three blocks, were delivered within each experimental session. Depending on the experimental block (see the experimental procedure), the laser intensity $\left(\mathrm{mJ} / \mathrm{mm}^{2}\right)$ was set either at $3 \mathrm{~mJ} / \mathrm{mm}^{2}$ below or at 1.5 or $6 \mathrm{~mJ} / \mathrm{mm}^{2}$ above pain threshold.

\section{Experimental procedure}

After assembling the EEG electrodes, participants were then prepared for the evoked-potentials session, which involved a calibration phase and the familiarization with the experiment. They sat approximately $50 \mathrm{~cm}$ far from a 15 inches screen (Philips CRT) where visual stimuli were centrally shown using Presentation ${ }^{\circledR}$ software (Version 0.70, www.neurobs.com). Participants were asked to place their dominant hand on a desk and were instructed to watch the screen for the whole length of the experiment in order to follow the instructions. They were also asked to avoid eye movements. In the calibration phase, pain threshold was assessed using the method of limits (Gracely, 1994). Radiant laser pulses were delivered starting from sub-warm threshold $\left(1.5 \mathrm{~mJ} / \mathrm{mm}^{2}\right)$ until pain sensation was induced (pain threshold, T) with steps of $1.5 \mathrm{~mJ}$. The energy of the laser stimulus was individually set in order to elicit a pricking pain sensation which each subject reported verbally and that is related to the activation of A $\delta$ nociceptors (Treede et al., 1995). After establishing the pain threshold $\mathrm{T}$, the experiment started and a total of 72 painful and non painful stimuli, divided in three separate blocks (24 stimuli for each block), were delivered during the video presentation. The 
presentation was designed as follow: after an asterisk indicating the fixation point, a sign (+ or -) was presented in the center of the screen and then a picture with random lines appeared as a warning of the incoming laser pulse. Finally a sentence asking participants to rate the stimulus (from 0 to 10) appeared on the screen (Fig. 1A).

\section{Conditioning manipulation and experimental groups}

Participants received and rated three blocks of laser stimuli: two acquisition blocks (blocks 1 and 2) and 1 evocation block (block 3). In the acquisition blocks, + and - cues (CS) were paired respectively with increase $\left(\mathrm{T}+6 \mathrm{~mJ} / \mathrm{mm}^{2}\right)$ and decrease $\left(\mathrm{T}-3 \mathrm{~mJ} / \mathrm{mm}^{2}\right)$ of laser intensity (US). These blocks were aimed at associating the visual information with the increase/decrease of stimulus intensity. 12 stimuli associated with a + and 12 with a - sign were delivered in each block, thus, a total of 48 CS-US pairings were delivered in the acquisition phase. In the first two blocks, stimuli associated with + were above the pain threshold (more painful) and stimuli associated with - below the pain threshold (non painful). During the evocation block, pain intensity was the same $(\mathrm{T}+1.5$ $\mathrm{mJ} / \mathrm{mm}^{2}$ ), irrespective of the cue, and 12 stimuli were associated with a + sign and 12 with a - sign (Fig.1B).

Participants were randomly assigned to two different groups: 1) conditioning with verbal information group (VER, $\mathrm{N}=17$ ) or 2) conditioning without verbal information group (noVER, $\mathrm{N}=17$ ). The VER group was informed that during the whole experiment (acquisition and evocation phase) the + cue anticipated more painful stimulations, whereas the - cue anticipated less painful stimulations. Thus, the VER group received explicit verbal expectations about the meaning of the cues. Conversely, no information was provided to the noVER group participants as to the meaning of the + and - signs.

\section{Psychophysics}


To measure the subjective pain sensation after each laser pulse, participants were asked to verbally rate their pain experience according to a numerical rating scale (NRS) from 0 (no pain sensation) to 10 (maximal pain sensation). Before the actual experiment, the NRS was explained to each participant to check their understanding of the scale.

\section{EEG data analysis}

Pre-processing

EEG data were pre-processed and analyzed using Matlab (Mathworks Inc., Natick, Massachusetts, USA), EEGLAB (Delorme and Makeig, 2004) and Letswave (http://amouraux.webnode.com) (Mouraux and Iannetti, 2008). EEG data of each block were segmented into 24 epochs using a time window ranging from $0.5 \mathrm{~s}$ before to $1.5 \mathrm{~s}$ after the laser trigger (total epoch duration: $2 \mathrm{~s}$ ). Each epoch was baseline corrected using the pre-stimulus interval from $-500 \mathrm{~ms}$ to $0 \mathrm{~ms}$ as reference. The baseline correction was done by subtracting the mean of the signal of the pre-stimulus. EEG epochs were band-pass filtered from 1 to $30 \mathrm{~Hz}$ using the Fast Fourier Transformation filter. EOG artifacts were subtracted using a validated method based on independent component analysis (ICA) (Jung et al, 2000). In all datasets, ICs related to eye movements had a large EOG channel contribution reflected by a large frontal scalp distribution. A mean of $4 \pm 0.72$ ICs were removed in both groups. Since frequency filtering and ICA might have changed the EEG signals, a second baseline correction was performed using the same $-500 \mathrm{~ms}$ to $0 \mathrm{~ms}$ reference interval, thus ensuring that the average signal in the pre-stimulus interval was equal to 0 (Valentini et al., 2011; Ronga et al., 2012; Torta et al., 2012). Finally, epochs with amplitude values exceeding $\pm 75 \mu \mathrm{V}$ were rejected. These epochs constituted the $2.5 \%$ of the total number of epochs.

\section{LEP Analysis}

Trials were grouped depending on the cue preceding the laser stimulus (+ or -) and epochs preceded by the same cue were further low-pass filtered (high edge:30 Hz) and then averaged together, time- 
locked to the onset of laser stimulation. Thus, for each participant we obtained two averages, one corresponding to responses to laser stimuli after the + cue (more painful responses), the other to the response after the - cue (less painful response). The N2-P2 component, a biphasic negative-positive complex, was identified at the vertex $(\mathrm{Cz})$. The $\mathrm{N} 2$ was defined as the most negative deflection after stimulus onset, at approximately $230 \mathrm{~ms}$ after the trigger. The P2 wave was defined as the most positive deflection after stimulus onset, at approximately $150 \mathrm{~ms}$ after N2. Data are presented as a peak-to-peak measure.

\section{Statistical analysis}

Differences in NRS scores and LEP amplitudes were tested by means of a 2-way mixed ANOVA with cue '+ vs -' as within factor and group 'VER vs noVER' as between factor. A 3-way mixed ANOVA was used to test the effectiveness of the acquisition phase, with cue and block as within factors and group as between factor. Post-hoc Student-Neumann-Keuls was applied for multiple comparisons. Data are presented as mean \pm standard deviation (SD), and the level of significance was set at $\mathrm{P}<0.05$.

\section{Results}

VER and noVER groups did not differ in age $(\mathrm{P}=0.8)$ or gender $(\chi 2(1)=0.000, \mathrm{P}=1)$. No differences were observed in pain thresholds and pain threshold intensity ratings.

\section{Psychophysics}

In the acquisition phase, mean NRS scores after cue presentation in the VER group were $4.6 \pm 1.5$ for + and $0.7 \pm 0.7$ for - in block 1 , and $4.3 \pm 1.5$ for + and $0.5 \pm 0.6$ for - in block 2 . In the noVER group, mean NRS scores after cue presentation were $3.9 \pm 1.4$ for + and $0.7 \pm 0.8$ for - in block 1 , and $3.4 \pm 1.6$ for + and $0.6 \pm 0.8$ for - in block 2 . In the evocation block, mean NRS scores after cue 
presentation were $2.9 \pm 1.5$ for + and $2.3 \pm 1.2$ for - in the VER group, and $2.1 \pm 1.5$ for + and $2.1 \pm$ 1.6 for - in the noVER group.

As a first step, we verified that in the acquisition blocks, stimuli preceded by + were perceived as more intense than stimuli preceded by - cues. The results of the 3-way mixed ANOVA revealed a main effect of cue $(\mathrm{F}(1,32)=202.5, \mathrm{P}<0.001)$ which means that NRS scores were higher when preceded by $\mathrm{a}+$. We also found a trend in the cue $\mathrm{x}$ group interaction $(\mathrm{F}(1,32)=3.23, \mathrm{P}=0.078)$. Post-hoc tests highlighted that the trend was driven by a significant increase in pain rating after + cue presentations in the VER group compared with pain rating after + cue presentations the noVER group $(\mathrm{MSE}=1.265, \mathrm{df}=61.29, \mathrm{q}=2.58, \mathrm{P}<0.05)($ Fig.2 $)$.

Then we focused on the evocation block. The results of the 2-way mixed ANOVA showed a significant interaction in cue $\mathrm{x}$ group $(\mathrm{F}(1,32)=5.9, \mathrm{P}<0.02)$, indicating that the effect of cue on pain rating differed between the groups. Subsequent post-hoc comparisons revealed a significant decrease in NRS scores after - presentations in VER group (MSE $=2.126$, $\mathrm{df}=36.67, \mathrm{q}=1.82, \mathrm{P}<$ 0.002) but no differences in the noVER group (Fig.3).

\section{LEP amplitude}

All participants showed a clear-cut biphasic N2-P2 response. In the evocation block, the mean peak-to-peak amplitude was $19.6 \pm 8.5 \mu \mathrm{V}$ after + cue presentations and $16.6 \pm 8.5 \mu \mathrm{V}$ after cue presentations in the VER group, and respectively $20.7 \pm 13.7 \mu \mathrm{V}$ and $20.9 \pm 15.2 \mu \mathrm{V}$ in the noVER group (Fig. 4). The results of the 2-way mixed ANOVA showed a significant cue x group effect $(\mathrm{F}(1,32)=4.5, \mathrm{P}<0.05)$, indicating that the effect of cue on LEP amplitude differed between the groups. Again, post-hoc results showed a significant difference in LEP amplitude between + and - cues in the VER group (MSE $=141.24, \mathrm{df}=34.29, \mathrm{q}=-1.03, \mathrm{P}<0.01$ ) and no differences in the noVER group (Fig. 5). Moreover, two separate ANOVAs were carried out on N2 and P2 peak amplitudes in order to investigate the effect of cue on the single N2 and P2 components. The results showed a significant cue $x$ group effect $(\mathrm{F}(1,32)=8.5, \mathrm{P}<0.001)$ in $\mathrm{P} 2$ peak amplitudes, indicating 
that the effect of cue on P2 amplitude differed between the groups. Again, post-hoc tests showed a significant difference in P2 amplitude between + and - cue in the VER group $(\mathrm{MSE}=27.065$, $\mathrm{df}=$ 35.2, $\mathrm{q}=-1.88, \mathrm{P}<0.001)$ only, whereas no differences were present in the noVER group. No differences were observed for the N2 component.

\section{Discussion}

The aim of the present study was to investigate the role of cognitive factors in conditioned analgesia. To do this, we used a well-established conditioning procedure in which 48 laser pulses were paired with a visual cue that corresponded to either an increase $(+)$ or decrease $(-)$ in stimulus intensity. The two groups of healthy subjects differed only for the verbal information provided at the beginning of the experiment: the VER group was informed that the + cue presentations anticipated more painful stimuli compared with the - ones. Conversely, the noVER group did not receive any explicit information about the meaning of the + and - cues. This difference in verbal information turned out to be crucial in producing a conditioned analgesic response: only the VER group reported a modulation of pain perception in the evocation phase, and this occurred at both the psychophysical and at the neurophysiological level.

At least two important points need to be emphasized. First, according to cognitive theories of conditioning, it is unlikely that the US-CS temporal contiguity alone is the only factor involved in conditioning process (Rescorla, 1988; Fanselow, 1998). Cognitive elements such as surprise (Kamin et al., 1968), expectation (Montgomery and Kirsch, 1997), motivation and emotions (Reiss, 1980; Rescorla, 1988; Kirsch et al., 2004) have been postulated to play a major role in the conditioning process. In line with these theories, our results indicate that, at least in some circumstances, just pairing a cue with different pain stimulus intensities is not sufficient per se to produce a learning process. What matters is the explicit knowledge about the meaning assigned to the visual cue. Similar results have been documented in nicotine conditioning studies where it has been shown that cognitive factors, such as explicit nicotine reward knowledge, are necessary in the 
establishment of addictive behaviors (Hogarth and Duka, 2006).

Second, the explicit information provided at the beginning of the experiment seems to be a key element in the learning process, namely, in the acquisition phase. For example, Mongomery and Kirsch (1997) used a design in which two groups were conditioned with the surreptitious reduction of pain intensity (US) in the presence of an inert cream (CS) that they believed to be effective. One conditioning group was informed that stimulus intensity level was reduced during the placebo trials, while the other conditioning group was not informed about this reduction. Indeed, in the following evocation phase, conditioned analgesia occurred only in the latter, uninformed, group. Moreover, in a more recent study it has been shown that, after a pharmacological preconditioning with ketorolac in which verbal instructions about analgesia were delivered (acquisition phase), no conditioned analgesia occurred when ketorolac was replaced with a placebo along with the opposite suggestion of hyperalgesia (evaluation phase) (Benedetti et al., 2003).

Our study underscores the crucial influence of explicit verbal information during both the acquisition and evaluation phase.

From a neurophysiological point of view, our results are consistent with the psychophysical data, since no difference between the + and - condition was found in the noVER group. Conversely, the results in the VER group are consistent with those of previous studies that reported a modulation of the N2-P2 complex after a conditioning procedure (Colloca et al., 2008; Wager et al., 2006; Watson et al., 2006). In particular, placebo responses have been found to be associated to a reduction of the amplitude of the biphasic N2-P2 vertex component. In line with our results, Wager et al. (2006) reported that a placebo treatment decreased the amplitude of the P2 component, and Colloca et al. (2008) reported a decrease of peak-to-peak amplitude after placebo-related expectation. Likewise, Watson et al. (2007) found that the decrease in LEP amplitude was related to both the N2 and P2 components. Although the functional significance of LEP remains to be fully understood (Iannetti et al., 2008; Ronga et al., 2012; Torta et al., 2012; Valentini et al., 2011), LEP are often considered the best tool for investigating the nociceptive system (Cruccu and García- 
Larrea, 2004) for their selectivity in the activation of A-delta and C nociceptive fibers and their correlation with the perceived intensity of painfulness (Iannetti et al., 2005). LEPs represent the sum of neural activities arising from several cortical generators partly localized using dipole modeling of scalp and intracerebral recordings [for a review, see Garcia-Larrea et al., 2003]. The N2-P2 complex seems to originate in bilateral operculo-insular cortices and in the cingulate gyrus and is modulated by top-down processes (Lorenz and Garcia-Larrea, 2003) and bottom-up mechanisms (Legrain et al., 2012).

In previous studies that used a similar experimental approach, two opposite conclusions were reached. Voudouris et al. $(1985 ; 1990)$ associated a non-anesthetic cream (placebo) to the surreptitious reduction of the intensity of painful stimulation, so as to make the subjects believe that the cream was an effective anesthetic. These subjects, who had experienced a "true anesthesia/analgesia”, became strong placebo responders, which suggests that conditioning is important. However, expectation was found to be crucial, because no placebo analgesic effect was found if the subjects were told about the manipulation of intensities (Montgomery and Kirsch, 1997). This suggests that, during a conditioning procedure, conscious expectations of a future outcome play a major role. However, expectation and conditioning are not necessarily mutually exclusive, as they may represent two sides of the same coin. In other words, a conditioning procedure might lead to conditioned placebo responses through a mechanism of "reinforced expectations”. Indeed, in the 1960s, a different interpretation of classical conditioning was put forward (Rescorla, 1968; Kamin, 1969). According to this reinterpretation, conditioning does not depend merely on the pairing of conditioned and unconditioned stimuli, but on the cognitive information of the conditioned stimulus. Therefore, a conditioning procedure would lead to the expectation that a given event will follow another event (Reiss, 1980; Rescorla, 1988; Kirsch et al., 2004). Our data are in line with a recent work on itch, where no placebo and nocebo effect were reported after a conditioning procedure without verbal suggestion (Bartels et al., 2014). 
Even if expectations play a crucial role in the conditioning processes, conditioning is not a unitary mechanism and expectations have no effects when unconscious physiological functions are involved, such as hormonal secretion (Benedetti et al., 2003). Moreover, conditioned pain perception without verbal instruction concerning the meaning of the CS has been shown in different studies (Büchel et al., 1998; Jensen et al., 2012; Waschulewski-Floruss et al. 1994). However, it is noteworthy that there are important differences in the experimental procedures, such as the number of CS-US pairings, the temporal contingency between CS and US, the duration of the painful stimulation and the type of CS involved. For example, some of these studies (Büchel et al., 1998; Jensen et al., 2012) used faces as CS stimuli, which have a specific brain representation (e.g. Vuilleumier et al., 2001; van de Riet et al., 2009), differently from our CSs.

Our results support the notion that conditioned analgesia requires cognitive processes through which the information and the meaning of the conditioned stimulus are key factors during the learning process. In other words, the mere pairing between a visual cue and pain reduction is not sufficient to generate a conditioned analgesic response in humans. These experimental data may have important implications in clinical practice, for they show that verbal communication and interaction between health professionals and patients are essential elements in the therapeutic outcome.

\section{Author Contributions}

Elisa Carlino: conception and design, acquisition of data, analysis and interpretation of data, drafting the article

Diana Torta: analysis and interpretation of data, drafting the article

Alessandro Piedimonte: analysis and interpretation of data, drafting the article

Elisa Frisaldi: acquisition of data, drafting the article

Sergio Vighetti: acquisition of data, discussion of results

Fabrizio Benedetti: conception and design, drafting the article and critically revised 


\section{References}

Ader R (1997). The role of conditioning in pharmacotherapy. In: A Harrington, ed. The Placebo Effect: An Interdisciplinary Exploration, pp. 138-65. Harvard University Press, Cambridge, MA.

Arntz, A. (1996). Why do people tend to overpredict pain? On the asymmetries between underpredictions and overpredictions of pain. Behav. Res. Ther., 34, 545554.

Atlas, L. Y., Whittington, R. A., Lindquist, M. A., Wielgosz, J., Sonty, N., \& Wager, T. D. (2012). Dissociable Influences of Opiates and Expectations on Pain. J. Neurosci., 32, 80538064.

Bartels, D. J. P., van Laarhoven, A. I. M., Haverkamp, E. A., Wilder-Smith, O. H., Donders, A. R. T., van Middendorp, H., ... Evers, A. W. M. (2014). Role of Conditioning and Verbal Suggestion in Placebo and Nocebo Effects on Itch. PLoS ONE, 9, e91727.

Benedetti, F., Pollo, A., Lopiano, L., Lanotte, M., Vighetti, S., and Rainero, I. (2003). Conscious expectation and unconscious conditioning in analgesic, motor, and hormonal placebo/nocebo responses. J. Neurosci., 23, 43154323.

Bromm, B., and Treede, R.D. (1984). Nerve fibre discharges, cerebral potentials and sensations induced by CO2 laser stimulation. Hum. Neurobiol., 3, 3340.

Büchel, C., Morris, J., Dolan, R. J., \& Friston, K. J. (1998). Brain systems mediating aversive conditioning: an event-related fMRI study. Neuron, 20, 947957. 
Bushnell, M.C., Čeko, M., and Low, L.A. (2013). Cognitive and emotional control of pain and its disruption in chronic pain. Nat. Rev. Neurosci., 14, 502511.

Carlino, E., Pollo, A., and Benedetti, F. (2011). Placebo analgesia and beyond: a melting pot of concepts and ideas for neuroscience. Curr Opin Anaesthesiol 24, 540544.

Colloca, L., Tinazzi, M., Recchia, S., Le Pera, D., Fiaschi, A., Benedetti, F., and Valeriani, M. (2008). Learning potentiates neurophysiological and behavioral placebo analgesic responses. Pain, 139, 306-314.

Cruccu, G., and García-Larrea, L. (2004). Clinical utility of pain--laser evoked potentials. Suppl. Clin. Neurophysiol., 57, 101110.

Delorme, A., and Makeig, S. (2004). EEGLAB: an open source toolbox for analysis of single-trial EEG dynamics including independent component analysis. J. Neurosci. Methods, 134, 921.

Fanselow, M.S. (1998). Pavlovian conditioning, negative feedback, and blocking: mechanisms that regulate association formation. Neuron, 20, 625627.

Garcia-Larrea, L., Frot, M., and Valeriani, M. (2003). Brain generators of laser-evoked potentials: from dipoles to functional significance. Neurophysiol. Clin., 33, 279292.

Gracely RH (1994) Studies of pain in normal man. In: Wall P, Melzack R (eds) Textbook of pain 3rd edn. Churchill Livingstone, New York. 
Guo, J.-Y., Wang, J.-Y., and Luo, F. (2010). Dissection of placebo analgesia in mice: the conditions for activation of opioid and non-opioid systems. J. Psychopharmacol., 24, 15611567.

Harris, J.A., and Westbrook, R.F. (1996). Midazolam impairs the acquisition of conditioned analgesia if rats are tested with an acute but not a chronic noxious stimulus. Brain Res. Bull., 39, 227233.

Hogarth, L., and Duka, T. (2006). Human nicotine conditioning requires explicit contingency knowledge: is addictive behaviour cognitively mediated? Psychopharmacol, 184, 553566.

Iannetti, G.D., Hughes, N.P., Lee, M.C., and Mouraux, A. (2008). Determinants of laser-evoked EEG responses: pain perception or stimulus saliency? J. Neurophysiol. 100, 815828. Iannetti, G.D., Niazy, R.K., Wise, R.G., Jezzard, P., Brooks, J.C.W., Zambreanu, L., Vennart, W., Matthews, P.M., and Tracey, I. (2005). Simultaneous recording of laser-evoked brain potentials and continuous, high-field functional magnetic resonance imaging in humans. Neuroimage, 28, 708719. Interdisciplinary Research Agenda, pp. 133 57. BMJ Books, London.

Jensen, K. B., Kaptchuk, T. J., Kirsch, I., Raicek, J., Lindstrom, K. M., Berna, C., ... Kong, J. (2012). Nonconscious activation of placebo and nocebo pain responses. PNAS, 109, 1595915964.

Jung, T.P., Makeig, S., Westerfield, M., Townsend, J., Courchesne, E., and Sejnowski, T.J. (2000). Removal of eye activity artifacts from visual event-related potentials in normal and clinical subjects. Clin. Neurophysiol., 111, 17451758. 
Kamin, L.J. (1968). “Attention-like” processes in classical conditioning. In M.R. Jones (Ed.). Miami Symposium on the Prediction of Behavior, 1967: Aversive Stimulation. (Coral Gables, Florida: University of Miami Press) pp. 9-31.

Kamin, L.J. (1969). Predictability, surprise, attention, and conditioning. In B. A. Campbell and R. M. Church (Eds.) Punishment and Aversive Behavior. New York: Appleton-Century-Crofts.

Kirsch, I., Lynn, S.J., Vigorito, M., and Miller, R.R. (2004). The role of cognition in classical and operant conditioning. J. Clin. Psychol., 60, 369392.

Legrain, V., Mancini, F., Sambo, C.F., Torta, D.M., Ronga, I., and Valentini, E. (2012). Cognitive aspects of nociception and pain: bridging neurophysiology with cognitive psychology. Neurophysiol. Clin. 42, 325336.

Lorenz, J., and Garcia-Larrea, L. (2003). Contribution of attentional and cognitive factors to laser evoked brain potentials. Clin. Neurophysiol. 33, 293301.

Lui, F., Colloca, L., Duzzi, D., Anchisi, D., Benedetti, F., and Porro, C.A. (2010). Neural bases of conditioned placebo analgesia. Pain, 151, 816824.

Matzel, L.D., and Miller, R.R. (1987). Recruitment time of conditioned opioid analgesia. Physiol. Behav., 39, 135140.

Montgomery, G.H., and Kirsch, I. (1997). Classical conditioning and the placebo effect. Pain 72, 107113. 
Mouraux, A., and Iannetti, G.D. (2008). Across-trial averaging of event-related EEG responses and beyond. MRI, 26, 1041 1054. New York.

Pavlov, I.P. (1927). Conditioned Reflexes: An Investigation of the Physiological Activity of the Cerebral Cortex (Oxford University Press).

Reiss, S. (1980). Pavlovian conditioning and human fear: An expectancy model. Behavior Therapy 11, 380396.

Rescorla, R. A. (1968). Probability of shock in the presence and absence of CS in fear conditioning. J. Comp. Physiol. Psychol., 66, 15.

Rescorla, R.A. (1988). Pavlovian conditioning: It's not what you think it is. American Psychologist 151160.

Ronga, I., Valentini, E., Mouraux, A., and Iannetti, G.D. (2012). Novelty is not enough: laserevoked potentials are determined by stimulus saliency, not absolute novelty. J. Neurophysiol., 109, 692701.

Ross, R.T. (1986). Pavlovian second-order conditioned analgesia. J. Exp. Psychol. Anim. Behav. Process, 12, 3239.

Siegel S (2002). Explanatory mechanisms for placebo effects: Pavlovian conditioning. In: HA Guess, A Kleinman, JW Kusek, LW Engel, eds. The Science of the Placebo: Toward an Interdisciplinary Research Agenda, H.A. Guess, A. Kleinman, J.W. Kusek, L.W. Engel, eds. (London: BMJ Books) pp. 133-157. 
Tolman EC (1932). Purposive Behavior in Animals and Men. Appleton Century Crofts, New York.

Torta, D.M., Liang, M., Valentini, E., Mouraux, A., and Iannetti, G.D. (2012). Dishabituation of laser-evoked EEG responses: dissecting the effect of certain and uncertain changes in stimulus spatial location. Exp. Brain Res., 218, 361372.

Treede, R.D., Meyer, R.A., Raja, S.N., and Campbell, J.N. (1995). Evidence for two different heat transduction mechanisms in nociceptive primary afferents innervating monkey skin. J. Physiol., 483 ( Pt 3), 747758.

Truini, A., Galeotti, F., Romaniello, A., Virtuoso, M., Iannetti, G.D., and Cruccu, G. (2005). Laserevoked potentials: normative values. Clin. Neurophysiol., 116, 821826.

Valentini, E., Torta, D.M.E., Mouraux, A., and Iannetti, G.D. (2011). Dishabituation of laserevoked EEG responses: dissecting the effect of certain and uncertain changes in stimulus modality. J. Cogn. Neurosci., 23, 28222837.

Van de Riet, W. A. C., Grezes, J., \& de Gelder, B. (2009). Specific and common brain regions involved in the perception of faces and bodies and the representation of their emotional expressions. Social. Neurosci., 4, 101120.

Villemure, C., and Bushnell, M.C. (2002). Cognitive modulation of pain: how do attention and emotion influence pain processing? Pain 95,195 199.

Villemure, C., and Bushnell, M.C. (2009). Mood Influences Supraspinal Pain Processing Separately from Attention. J. Neurosci., 29,705 715. 
Voudouris, N.J., Peck, C.L., and Coleman, G. (1985). Conditioned placebo responses. J. Pers. Soc. Psychol., 48, 4753.

Voudouris, N.J., Peck, C.L., and Coleman, G. (1990). The role of conditioning and verbal expectancy in the placebo response. Pain, 43, 121128.

Vuilleumier, P., Armony, J. L., Driver, J., \& Dolan, R. J. (2001). Effects of Attention and Emotion on Face Processing in the Human Brain: An Event-Related fMRI Study. Neuron, 30, 829841.

Wager, T.D., Matre, D., and Casey, K.L. (2006). Placebo effects in laser-evoked pain potentials. Brain Behav. Immun. 20, 219230.

Waschulewski-Floruss, H., Miltner, W., Brody, S., \& Braun, C. (1994). Classical conditioning of pain responses. Int. J. Neurosci., 78, 2132.

Watson A., El-Dereby W., Vogt B.A, Jones A.K. (2007). Placebo analgesia is not due to compliance or habituation: EEG and behavioural evidence. Neurorep. 18, 771-5.

Watson, A., El-Deredy, W., Bentley, D.E., Vogt, B.A., and Jones, A.K.P. (2006). Categories of placebo response in the absence of site-specific expectation of analgesia. Pain 126, 115122.

Watson, A., El-Deredy, W., Iannetti, G.D., Lloyd, D., Tracey, I., Vogt, B.A., Nadeau, V., and Jones, A.K.P. (2009). Placebo conditioning and placebo analgesia modulate a common brain network during pain anticipation and perception. Pain 145, 2430. 
Wickramasekera, D.I. (1980). A conditioned response model of the placebo effect. Biofeedback Self. Regul., 5, 518. 


\section{Figure legends}

Figure 1. Experimental design. A) Participants were instructed to follow a video presentation during the experiment. The presentation was designed as follow: after an asterisk indicating the fixation point, a sign ( + or - ) was presented in the center of the screen and then a picture with random lines appeared as a warning of the incoming laser pulse. Finally a sentence asking participants to rate the stimulus (from 0 to 10) appeared on the screen. B) Participants received three blocks of laser stimuli: two acquisition blocks and one evocation block. The intensity of stimulation was respectively lower (T-3 mJ/mm ${ }^{2}$ ) when - signs were presented (broken lines) and higher $\left(\mathrm{T}+6 \mathrm{~mJ} / \mathrm{mm}^{2}\right)$ when + signs were presented (black lines) in the acquisition blocks, so that participants experienced respectively real decrease and increase of their non painful and painful perception. In the evocation block the intensity was the same $\left(\mathrm{T}+6 \mathrm{~mJ} / \mathrm{mm}^{2}\right.$, gray lines), and 12 stimuli were paired with a + and 12 with a - sign.

Figure 2. Pain ratings in the acquisition blocks. A significant increase in pain perception was observed in the VER group (black) compared with noVER group (gray) when + cues where presented.

Figure 3. Pain ratings in the evocation blocks, when + and - cues were presented and the intensity of the laser stimulation was the same. A significant difference in pain rating was observed between + and - cues in the VER group, whereas no differences occurred in the noVER group.

Figure 4. Grand-average LEP acquired in $\mathrm{Cz}$ in the evocation block of the VER group (A) and noVER group (B) after - cue presentation (gray line) and + cue presentation (black line). Scalp maps show peak values in $\mathrm{Cz}$ in both groups. A significant difference in LEP amplitude was 
observed between the + and - cues in the VER group, whereas no differences occurred in the noVER group.

Figure 5. N2-P2 LEP amplitudes. A significant difference in N2-P2 peak-to-peak amplitude was observed between + and - cues in the VER group $(\mathrm{P}<0.01)$, whereas no differences were present in the noVER group (gray). 